\title{
A Prospective Study to Evaluate the Clinical Performance of A Novel Multitarget- Based Antigen Test on the Detection of Sars-Cov-2 in Symptomatic Population- Comparison to Nuclear Acid-Based Tests
}

\author{
Weibo $\mathrm{Yu}^{1}$, Elizabeth Rao' ${ }^{1}$, Daisy Defang Wan ${ }^{2}$, Chris Patterson ${ }^{2}$, Dylan Delong Wan ${ }^{2}$, Arutyun Pogosyan², John Wan ${ }^{2}$, and \\ Jianyu Rao ${ }^{1, *}$
}

${ }^{1}$ Department of Pathology and Laboratory Medicine, David Geffen School of Medicine, University of California, Los Angeles, California, USA ${ }^{2}$ W.H.P.M., Inc., Los Angeles, California, USA

*Corresponding authors: Jianyu Rao, Department of Pathology and Laboratory Medicine, University of California, Los Angeles, California, USA, E-mail: Jrao@mednet.ucla.edu

Received: 17 Mar, 2021 | Accepted: 05 Apr, 2021 | Published: 16 Apr, 2021

Citation: Yu W, Rao E, Wan DD, Patterson C, Wan DD, et al. (2021) A Prospective Study to Evaluate the Clinical Performance of A Novel Multitarget-Based Antigen Test on the Detection of Sars-Cov-2 in Symptomatic Population-Comparison to Nuclear Acid-Based Tests. J Epidemiol Public Health Rev 6(1): dx.doi.org/10.16966/2471-8211.211

Copyright: (C) $2021 \mathrm{Yu} \mathrm{W}$, et al. This is an open-access article distributed under the terms of the Creative Commons Attribution License, which permits unrestricted use, distribution, and reproduction in any medium, provided the original author and source are credited.

\begin{abstract}
The study was designed to evaluate the clinical performance characteristics of the First Sign ${ }^{\circledR}$ SARS-CoV- 2 Antigen Test, a visual read lateral flow immunoassay (W.H.P.M., Inc.). Individuals presenting at the study sites were evaluated by health care providers for COVID-19 symptoms and sequentially enrolled. The standard of care nasopharyngeal swab sample was first collected for RT-PCR testing. Participant self-collection nasal swab sample was then obtained for the Antigen Test. The clinical performance characteristics of the study device were calculated by comparing participant study device test result recorded at the point of care with the participant matched RT-PCR results provided by qualified CLIA high complexity laboratories. A total of 128 symptomatic adult participants were included in the study, of which $46.8 \%$ (60/128) were determined to be RT-PCR positive for SARS-CoV-2. The mean comparator cycle threshold value (Ct) for RT-PCR positive participants included in the study was $\mathrm{Ct} 25$ (range $\mathrm{Ct}$ 18-Ct 39). The First Sign ${ }^{\oplus}$ SARS-CoV-2 Antigen Test demonstrated an overall clinical sensitivity of $96.7 \%$ (58/60) and a clinical specificity of $98.5 \%$ $(67 / 68)$ when compared with EUA RT-PCR comparator results for the detection of SARS-CoV-2 in symptomatic individuals within 5 days of symptom onset. The clinical sensitivity for samples with RT-PCR Ct<30 was $100 \%$ (48/48), 90\% (10/12) for samples with RT-PCR Ct $\geq 30$, and $98.2 \%$ (55/56) for samples with RT-PCR Ct $\leq 34$. Study results indicate that during the initial phase of infection, when upper respiratory viral loads and viral shedding levels are typically highest, an observed anterior nasal swab self-collected by the patient and applied to the First Sign ${ }^{\circledR}$ SARS-CoV- 2 Antigen Test may be as effective in detecting the presence of SARS-CoV-2 in symptomatic individuals as a EUA RT-PCR assay. Additionally, the ability to obtain a low-cost qualitative result within 15 minutes, combined with the simplicity of the anterior nasal self-sampling procedure, may dramatically increase testing efficiency while significantly reducing risk to health care providers by minimizing or eliminating the need for close contact with the patient.
\end{abstract}

Keywords: COVID-19; SARS-CoV-2; Antigen test; Clinical performance

\section{Introduction}

The COVID-19 pandemic, caused by severe acute respiratory syndrome coronavirus 2 (SARS-CoV-2), has to date infected approximately 100,000,000 individuals and resulted in over 2,000,000 deaths globally [1]. Current approach for the diagnosis of this RNA virus infection relies on nucleic acid amplification tests (NAATs). Reverse-transcription polymerase chain reaction (RT-PCR) molecular assay has been considered the gold standard for SARSCoV-2 diagnosis. Indeed, in acute respiratory infection, RT-PCR is routinely used to detect causative viruses from respiratory secretions [2]. However, with the rapid progress of the COVID-19 pandemic, the test approach using RT-PCR alone has demonstrated a number of limitations that hindered the control of this devastating pandemic. RT-PCR is time-consuming and relatively expensive, requiring 
adequate facilities and funding in order to keep up with the rapid spread of SARS-CoV-2. On the top of that, there is the need to perform the test in a well-controlled laboratory setting and to account for the long turnaround time of the test [3]. This proved difficult even for well-funded health care systems and led to delayed responses at the onset of the pandemic.

Recently, several SARS-CoV-2 antigen-based tests, which work via a lateral flow immunoassay mechanism, have been developed and received Emergency Use Authorization (EUA) from the United States Food and Drug Administration (FDA) [4-6]. Unlike serology assays, which detect the presence of the target antibodies that are developed in later stages of the body's immune response, antigen-based tests are capable of detecting the early onset of SARS-CoV-2 infection. The short turnaround time and one-step procedure of antigen tests may provide added value for rapid screening, patient triage, and case identification. The less expensive antigen-based tests may contribute to mass producibility when compared to RT-PCR, making rapid testing more readily available for lower- and middle-income countries. Antigen tests can also be used in high-risk congregate settings in which repeat testing could quickly identify persons with a SARS$\mathrm{CoV}-2$ infection to inform infection prevention and control measures, thus preventing transmission.

However, although rapid antigen tests generally have a satisfactory specificity (between $90 \%$ and $100 \%$ ), they demonstrated a lower diagnostic sensitivity in comparison to molecular testing, especially with specimens containing low viral loads [7]. The implementation and widespread usage of an antigen test should be verified under well-defined circumstances. First Sign ${ }^{\circ}$ SARS-CoV-2 Antigen Test was recently developed and manufactured by W.H.P.M., Inc. This unique test uses hybrid combination of monoclonal antibody pairs for multi-epitope targeting of the nucleocapsid protein in an effort to improve diagnostic sensitivity and retain specificity. We conducted a prospective study to investigate the clinical performance of this test on the detection of SARS-CoV-2 infection in a COVID-19 symptomatic population. Study device results were compared with two different standard of care FDA Emergency Use Authorized (EUA) RT-PCR tests to determine the negative percentage agreement (NPA), the positive percentage agreement (PPA), the positive percentage agreement relative to RT-PCR comparator cycle threshold values $(\mathrm{Ct})$, and the positive percentage agreement relative to symptom onset timeline.

\section{Methods}

\section{Study design and subject enrollment}

This is a prospective study to evaluate the clinical performance characteristics of the First Sign ${ }^{\star}$ SARS-CoV-2 Antigen Test. The study was carried out at two different sites at Los Angeles: Genx Laboratories (Point of Care Pandemic Response Site, Site A) and Angel Wings Medical Associates Urgent Care (Site B). Eligible participants presenting at the study sites between December 2020 and January 2021 were continuously screened and enrolled in the study. Eligible participants were defined as individuals presenting with symptoms commonly associated with COVID-19, reporting symptom onset $<6$ days prior to testing, capable of conducting self-nasal swab specimen collection, willing and able to provide informed consent, and undergoing RT-PCR sample collection. We excluded asymptomatic individuals and participants with "invalid" or "inconclusive" RT-PCR results. Patient age, gender, symptom onset time and exposure history information were collected. No study procedures were performed without an informed consent process or signature of a consent form. This research was performed in accordance with Good Clinical
Practice guidelines and the Declaration of Helsinki. This study was approved by Advarra Institute Review Board (Number: Pro00048222).

\section{Specimen collection and antigen test}

Two participant samples were consecutively collected from study participants. The standard of care sample was collected first by health care provider via nasopharyngeal swab eluted in Viral Transport Medium (VTM) in accordance with the Comparator Instructions for Use (IFU). Approximately 3 to 5 minutes post standard of care sample collection participants were provided with a copy of the study device specific Patient Quick Guide, an unopened nasal swab, and a study device sample collection tube containing $350 \mu \mathrm{L}$ of viral extraction buffer. Participants were instructed to follow the Patient Quick Guide step-by-step instructions and self-collect a nasal swab sample under the observation of the health care provider. As part of the step-by-step procedure, the swab was eluted, mixed, and sealed by the participant in the patient sample tube immediately after completing the self-nasal swab. The sealed patient sample was then returned to the operator for testing. Antigen test device and results determination are shown in figure 1 .

\section{RT-PCR test}

The two study sites utilized two different RT-PCR systems. Comparator device A (Site A): Applied Biosystems ${ }^{\mathrm{Tn}}$ Quant Studio $^{\mathrm{mn}} 7$ Pro Real-Time PCR/ Quidel Lyra ${ }^{\oplus}$ SARS-CoV-2 assay. Nasopharyngeal patient swabs eluted in VTM (Viraport Direct VTM $1.5 \mathrm{~mL}$ ) were tested in accordance with the IFU and laboratory SOP. Viral nucleic acid was extracted with the NucliSens ${ }^{\oplus}$ easy $\mathrm{MAG}^{\mathrm{max}}$ extraction system, and transferred to a 96-well plate for testing. Patient samples testing positive were repeated in duplicate for confirmation. Comparator Device B (Site B): Applied Biosystems ${ }^{\text {Tx }} 7500$ Real-Time PCR/ Quest SARS-CoV-2 RT-PCR assay. Nucleic acids were extracted from patient

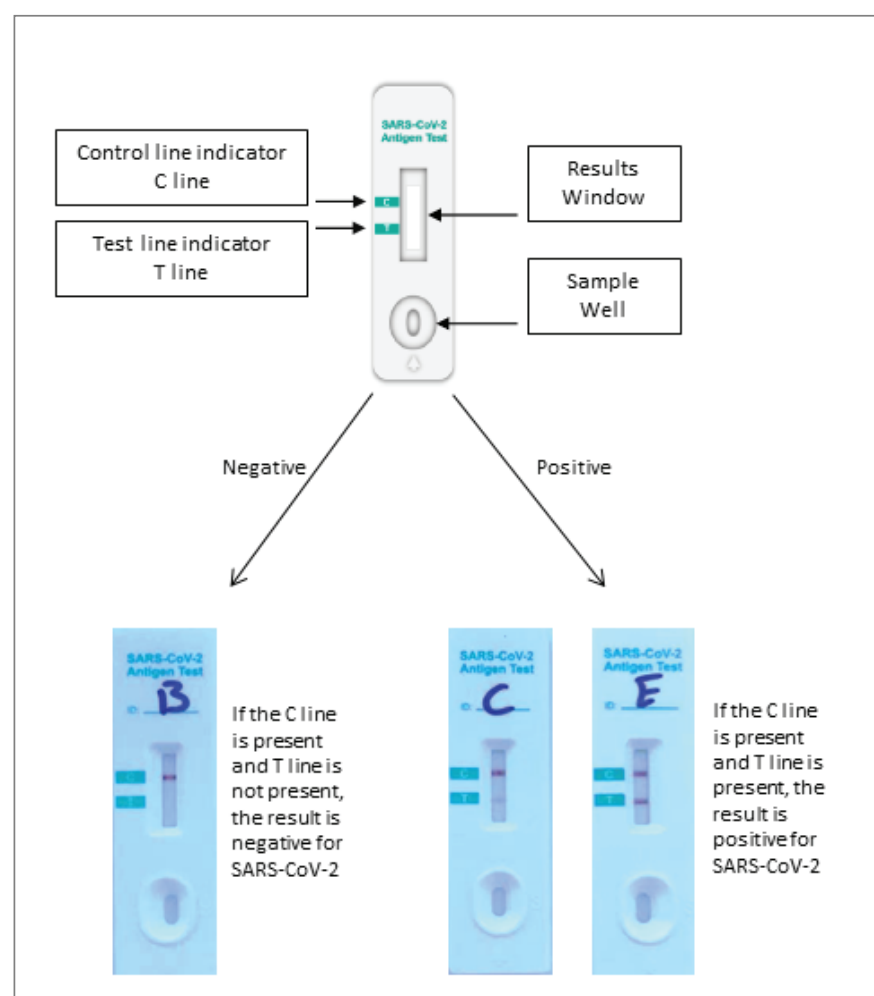

Figure 1: SARS-CoV-2 antigen test device and representative results. 
specimens stored in VTM using the Mag-Bind Viral RNA Xpress Kit (Omega Bio-Tek) on the Hamilton Microlab STAR Autoload automated platform. Post extraction, nucleic acids were transferred to a standard 96-well plate and tested according to the Quest Laboratory Standard Operation Procedure (SOP).

\section{Statistical analysis}

Data was entered and organized by Excel. Clinical performance analysis including clinical specificity (negative percentage agreement, or NPA), clinical sensitivity (positive percentage agreement, PPA), PPA relative to RT-PCR comparator cycle threshold values $(\mathrm{Ct})$, and PPA relative to symptom onset timeline, were calculated using RTPCR tests as the gold standard.

\section{Results}

\section{Study population and RT-PCR test results}

A total of 128 continuously enrolled symptomatic adults were included in the study. Overall study workflow is shown in figure 2. Samples from two participants were excluded due to inconclusive comparator results, and one sample was excluded due to procedural error. Participants testing RT-PCR positive for SARS-CoV-2 accounted for $46.8 \%(60 / 128)$ of the study cohort. Study participants were enrolled as they presented to the clinical study sites. Those enrolled represented a spectrum of socioeconomic backgrounds, ethnicities, and education levels. Females represented $68.2 \%$ of the study population (77/128), and $56.7 \%(34 / 60)$ of those testing RT-PCR positive for SARS-CoV-2. The median age of female participants was 48 years old with an age range of 19 to 77 years old. Male participants accounted for $39.8 \%$ $(51 / 128)$ of the study population and $44.3 \%(26 / 60)$ of the group that tested RT-PCR positive for SARS-CoV-2. The median age of male participants was 50 years old with an age range of 19 to 81 years old. In total, $44.1 \%$ (34/77) of all female participants included in the study tested RT-PCR positive for SARS-CoV-2, while 50.9\% (26/51) of male participants included in the study tested RT-PCR positive for SARSCoV-2 (Table 1).

Of the 60 RT-PCR positive results, $80 \%$ (48/60) were reported with a $\mathrm{Ct}<30$, and the remaining $20 \%(12 / 60)$ were reported as $\mathrm{Ct} \geq$ 30 , including $6.6 \%(4 / 60)$ with $\mathrm{Ct} \geq 35$. The mean Ct value for RTPCR positive participants included in the study was Ct 25 (range $\mathrm{Ct}$ 18-Ct 39) (Table 2). The distribution of RT-PCR Ct values relative to $\mathrm{Ct} 30$ is consistent with observations made by the FDA regarding the natural distribution of SARS-CoV-2 viral loads in a sequentially enrolled study population. The $\mathrm{Ct}$ values did not show a correlation with patient symptom onset time (Table 3 ).

\section{Clinical performance of antigen test}

The study device demonstrated an overall clinical sensitivity of $96.7 \%$ (58/60) and a clinical specificity of $98.5 \%$ (67/68) when compared with EUA RT-PCR comparator results for the detection of SARS-CoV-2 in symptomatic individuals (Table 4). The clinical sensitivity for samples with RT-PCR Ct<30 was 100\% (48/48), 90\% (10/12) for samples with RT-PCR Ct $\geq 30$, and $98.2 \%$ (55/56) for samples with RT-PCR Ct $\leq 34$. When disease prevalence is $2 \%$, the calculated PPV is $57.29 \%$ (95\% CI: $16.08 \%-90.38 \%$ ) and NPV is $99.93 \%$ (95\% CI: $99.73 \%-99.98 \%)$.

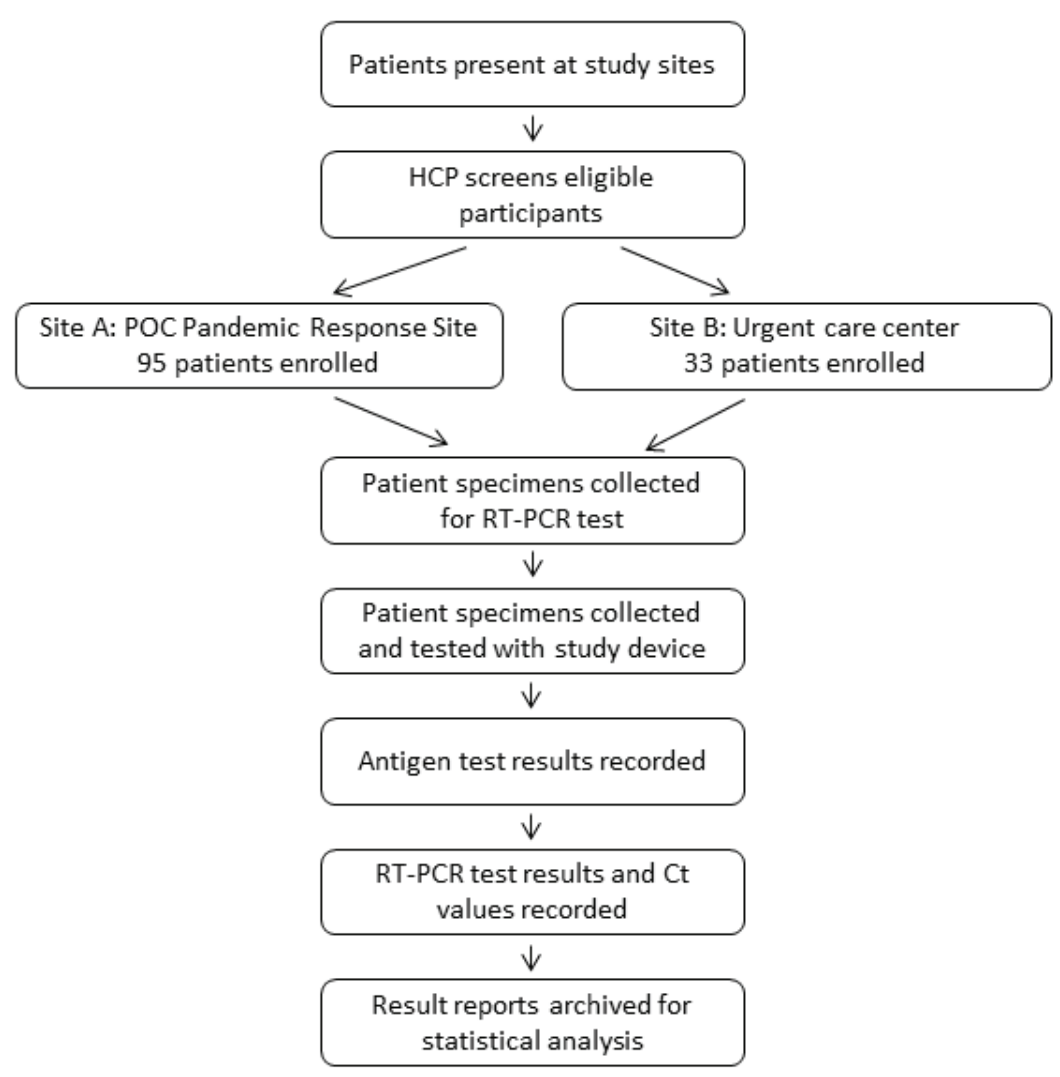

Figure 2: Study workflow overview. 
Table 1: Overview of participant characteristics.

\begin{tabular}{|c|c|c|c|c|c|c|c|}
\hline \multirow{2}{*}{\multicolumn{2}{|c|}{ Study Population }} & \multicolumn{2}{|c|}{ RT-PCR Comparator } & \multicolumn{4}{|c|}{ Study Device } \\
\hline & & POS [Total] & Prevalence & True POS [False NEG] & True NEG [False POS] & Sensitivity $(95 \% \mathrm{Cl})$ & Specificity $(95 \% \mathrm{Cl})$ \\
\hline \multirow{4}{*}{ Age Group } & $18-21$ & $2[7]$ & $28.5 \%$ & $2[0]$ & $5[0]$ & $100 \%(15.8 \%-100 \%)$ & $100 \%(47.8 \%-100 \%)$ \\
\hline & $22-30$ & $11[30]$ & $33.7 \%$ & $11[0]$ & $19[0]$ & $100 \%(71.5 \%-100 \%)$ & $100 \%(82.35 \%-100 \%)$ \\
\hline & $31-59$ & $36[74]$ & $47.7 \%$ & $34[2]$ & $37[1]$ & $94.4 \%$ (81.3\%-99.3\%) & $97.5 \%(86.2 \%-99.9 \%)$ \\
\hline & $60+$ & $11[17]$ & $64.7 \%$ & $11[0]$ & $6[0]$ & $100 \%$ (71.5\%-100\%) & $100 \%$ (54.1\%-100\%) \\
\hline \multirow{2}{*}{ Gender } & Female & 34 [77] & $44.2 \%$ & $32[2]$ & $43[0]$ & $94.1 \%(80.3 \%-99.3 \%)$ & $100 \%$ (91.8\%-100\%) \\
\hline & Male & $26[51]$ & $50.9 \%$ & $26[0]$ & $24[1]$ & $100 \%$ (86.8\%-100\%) & $96.0 \%(79.6 \%-99.9 \%)$ \\
\hline
\end{tabular}

POS: Positive; NEG: Negative; $\mathrm{Cl}$ : Confidence Interval

Table 2: Comparator cycle threshold value distribution.

\begin{tabular}{|c|c|c|c|c|c|c|c|}
\hline \multirow{3}{*}{ Study Site } & \multicolumn{7}{|c|}{ RT-PCR Comparator } \\
\hline & \multicolumn{2}{|c|}{$\mathrm{Ct}<30$} & \multicolumn{2}{|c|}{$\mathrm{Ct} \geq \mathbf{3 0}$} & \multirow{2}{*}{ Total POS } & \multirow{2}{*}{ RT-PCR NEG } & \multirow{2}{*}{ Total Samples } \\
\hline & Results & $\%$ of Total & Results & $\%$ of Total & & & \\
\hline Site A & 35 & $77.80 \%$ & 10 & $22.20 \%$ & 45 & 50 & 95 \\
\hline Site B & 13 & $86.70 \%$ & 2 & $13.30 \%$ & 15 & 18 & 33 \\
\hline Combined & 48 & $80.00 \%$ & 12 & $20.00 \%$ & 60 & 68 & 128 \\
\hline
\end{tabular}

Ct: Cycle threshold

Table 3: Symptom onset timeline and Ct value.

\begin{tabular}{|c|c|c|c|c|c|c|c|c|}
\hline \multirow{3}{*}{$\begin{array}{l}\text { Symptom Onset } \\
\text { Timeline }\end{array}$} & \multicolumn{7}{|c|}{$\begin{array}{l}\text { RT-PCR Comparator POS result } \\
\text { [Study device POS result] }\end{array}$} & \multirow{3}{*}{$\begin{array}{c}\text { Study device performance } \\
\text { PPA }(95 \% \mathrm{Cl})\end{array}$} \\
\hline & \multirow{2}{*}{$\mathrm{Ct}<30$} & \multirow{2}{*}{$30 \leq \mathrm{Ct}<35$} & \multirow{2}{*}{$\mathrm{Ct} \geq 35$} & \multicolumn{3}{|c|}{ Ct Value Distribution \% } & \multirow{2}{*}{ Mean Ct Value [Range] } & \\
\hline & & & & $\mathrm{Ct}<30$ & $30 \leq \mathrm{Ct}<35$ & $\mathrm{Ct} \geq 35$ & & \\
\hline Day 1 & $6[6]$ & $2[1]$ & $0[0]$ & $75 \%$ & $25 \%$ & $0 \%$ & $25[18-30]$ & $87.5 \%$ (47.4\%-99.7\%) \\
\hline Day 2 & $14[14]$ & $5[5]$ & $0[0]$ & $74 \%$ & $26 \%$ & $0 \%$ & $25[19-34]$ & $100 \%(71.5 \%-100 \%)$ \\
\hline Day 3 & $11[11]$ & $1[1]$ & $2[2]$ & $79 \%$ & $7 \%$ & $14 \%$ & $26[20-35]$ & $100 \%(76.8 \%-100 \%)$ \\
\hline Day 4 & $12[12]$ & $0[0]$ & $0[0]$ & $100 \%$ & $0 \%$ & $0 \%$ & 23 [20-29] & $100 \%$ (73.5\%-100\%) \\
\hline Day 5 & $5[5]$ & $0[0]$ & $2[1]$ & $71 \%$ & $0 \%$ & $29 \%$ & 29 [22-39] & $85.7 \%$ (42.1\%-99.6\%) \\
\hline Ct $<30$ Total & $48[48]$ & & & $80 \%$ & & & 23 [18-29] & $100 \%$ (92.6\%-100\%) \\
\hline $30 \leq \mathrm{Ct}<35$ Total & & $8[7]$ & & & $13 \%$ & & 32 [30-34] & $87.5 \%$ (47.4\%-99.7\%) \\
\hline $\mathrm{Ct} \geq 35$ Total & & & $4[3]$ & & & $7 \%$ & 36 [35-39] & $75.0 \%$ (19.4\%-99.4\%) \\
\hline Aggregate Ct Total & & $60[58]$ & & & $100 \%$ & & 25 [18-39] & $96.7 \%$ (88.5\%-99.6\%) \\
\hline
\end{tabular}

POS: Positive; Ct: Cycle threshold; PPA: Positive Percentage Agreement; $\mathrm{Cl}$ : Confidence Interval

\section{Discussion}

This rapid antigen test clinical performance validation study was conducted at a time in which the Los Angeles region encompassing both study sites underwent a dramatic increase of approximately 427,800 new COVID-19 cases within a 4-week time frame. During the same period, disease prevalence with the region was estimated to be as high as 10\%-20\% within Los Angeles County. Increasing test efficacy and generating timely results are critical. Low sensitivity is the main hurdle for the widespread usage of antigen test. Previously, Cochrane Diagnostic Test Accuracy Group summarized the varied sensitivity across all reported studies of rapid antigen test in August 2020. The average sensitivity was $56.2 \%$ (95\% CI 29.5 to $79.8 \%)$ and average specificity was $99.5 \%$ (95\% CI $98.1 \%$ to $99.9 \%$; based on 8 evaluations in 5 studies on 943 samples). Data from individual antigen tests were limited with no more than two studies for any test [8]. 
Table 4: Clinical performance characteristics of study device in comparison to RT-PCR.

\begin{tabular}{|c|c|c|c|c|c|}
\hline \multirow{2}{*}{\multicolumn{2}{|c|}{ Study Device Result }} & \multicolumn{2}{|c|}{ Comparator Result (RT-PCR) } & \multirow{2}{*}{ PPA $(95 \% \mathrm{Cl})$} & \multirow{2}{*}{ NPA $(95 \% \mathrm{Cl})$} \\
\hline & & \multirow{2}{*}{$\begin{array}{c}\text { Positive } \\
43\end{array}$} & \multirow{2}{*}{$\begin{array}{c}\text { Negative } \\
1\end{array}$} & & \\
\hline \multirow{2}{*}{ Site A } & Positive & & & \multirow{2}{*}{$95.6 \% 84.9 \%-99.5 \%$} & \multirow{2}{*}{$98.0 \% 89.4 \%-99.9 \%$} \\
\hline & Negative & 2 & 49 & & \\
\hline \multirow{2}{*}{ Site B } & Positive & 15 & 0 & \multirow{2}{*}{$100 \% 78.2 \%-100 \%$} & \multirow{2}{*}{$100 \% 81.5 \%-100 \%$} \\
\hline & Negative & 0 & 18 & & \\
\hline Clinical Sensitivity & & $58 / 60$ & & \multicolumn{2}{|c|}{$96.7 \%(88.5 \%-99.6 \%)$} \\
\hline Clinical Specificity & & & $67 / 68$ & \multicolumn{2}{|c|}{$98.5 \%$ (92.1\%-99.9\%) } \\
\hline
\end{tabular}

$\mathrm{Cl}$ : Confidence Interval

There is a growing demand for a rapid and accurate diagnostic test. Recently, more antigen test approaches have been developed and reported [9-11]. Roche SARS-CoV-2 rapid antigen test clinical verification study enrolled 321 consecutive patients with 149/321 (46.4\%) positive for SARS-CoV-2. The overall accuracy compared to molecular testing was $86.9 \%$, with $72.5 \%$ sensitivity and $99.4 \%$ specificity [12]. A recent rapid fluorescence immunoassays (FIAs) evaluation study tested 32 positive and 32 negative RT-PCR characterized clinical samples. Two assays detected $30(93.8 \%)$ and 29 (90.6\%) samples in RT-PCR positive group [13]. Another quantitative assay for detecting SARS-CoV-2 antigen was investigated in 100 nasopharyngeal samples collected from 47 SARS-CoV-2-infected patients. The sensitivity and specificity were $75.7 \%$ and $96.0 \%$, respectively [14]. While there seems to be a slightly improved sensitivity in detecting SARS-CoV-2 with these new generation of antigen tests, the overall sensitivity is still less than $80 \%$, which means over $20 \%$ infections may be missed by the test. One potential approach to overcome the low sensitivity of the antigen test is to utilize multiple antibody targets for the detection. This is the basis for WHPM's First Sign ${ }^{\circ}$ SARS-CoV-2 Antigen Test. WHPM's test is a lateral flow immunochromatographic membrane assay that applies a hybrid formulation of monoclonal antibodies to detect SARS-CoV-2 nucleocapsid protein from anterior nasal swab specimens, which potentially improves diagnostic sensitivity and retain the specificity. In this study, an overall clinical sensitivity of $96.7 \%$ and a clinical specificity of $98.5 \%$ were reported for the detection of SARS-CoV-2 in symptomatic individuals. This is comparable to EUA RT-PCR comparator when testing individuals within the first five days of symptom onset.

It is worth noting that most of antigen test studies demonstrated the sensitivity was highly correlated with the PCR cycle threshold value of the molecular test. The diagnostic value of the Panbio COVID-19 Ag Rapid Test (Abbott) was investigated in community-dwelling mildly symptomatic subjects. In community-dwelling subjects with mild respiratory symptoms, the Panbio COVID-19 Antigen Rapid Test had a sensitivity above $95 \%$ for nasopharyngeal samples when using Ctvalues $<32$ cycles as cut-off for RT-qPCR test positivity [15]. Another Roche SARS-CoV-2 rapid antigen study retrospectively tested 75 swabs from patients previously tested positive by SARS-CoV-2 PCR and 75 swabs from patients previously tested negative. The assay's sensitivity with samples with a cycle threshold of $<25$ and $25-<30$ was $100 \%$ and $95 \%$, respectively. However, the sensitivity dramatically reduced to $44.8 \%$ and $22.2 \%$ for samples with a cycle threshold of 30 $<35$ and $>35$, respectively [16]. A similar trend was observed in our study. In our study population, $80 \%$ of the RT-PCR positive patients with COVID-19 symptoms had a $<30$ cycle threshold. Currently, the clinical value of cycle threshold and correlation between cycle threshold value and severity of disease or mortality of patients are still debatable $[17,18]$. High sensitivity is important for massive population screening. Further studies are needed to investigate the detection of patients with low viral load. Interestingly, when using viral culture in determining the risk for potential transmissibility, in comparison to culture-positive results, the antigen test demonstrated a higher positive predictive value (90\%) than RT-PCR (70\%) [19]. Another viral infectivity study investigated antigen test for detecting infectious patients. Antigen tests demonstrated a more significant correlation with cell culture infectivity (61.8-82.4\%) compared to RT-PCR. These results indicated that rapid antigen test might be an alternative for screening potentially infective patients and reducing viral spread [20]. It should also be noted that both RT-PCR and antigen tests carry the risk of generating false-negative and false-positive results. Negative results may not exclude the possibility of SARS-CoV-2 infection. Patient clinical features need to be carefully reviewed for the management of COVID-19 pandemic.

Since the outbreak of the COVID-19 pandemic, several variants of SARS-CoV-2 have been identified in the UK, South Africa, and Brazil $[21,22]$. A latest study identified another novel variant of SARSCoV-2, CAL.20C in California, United States. This strain is defined by three mutations in the spike protein within a known receptor binding domain that has been found to be resistant to certain spike protein monoclonal antibodies $[23,24]$. The impact of these mutations on the body's immune response and the effectiveness of vaccines are being investigated. A reasonable concern is whether rapid antigen test could cover these mutations. The study device, with multiple monoclonal antibody pairs each targeting different epitopes of the nucleocapsid protein, may be capable of detecting these potentially dangerous mutated strains. Previous genome-wide analysis of SARS-CoV reported that the nucleocapsid gene is more stable and has acquired fewer mutations over time [25]. Immunoinformatic analysis of SARS$\mathrm{CoV}-2$ also demonstrated immune genic areas in nucleocapsid protein in the context of vaccine design [26,27]. Multi-epitope targeting of a well-conserved viral protein may be an effective method to mitigate the risk of lost sensitivity or false-negative results caused by viral mutations. However, additional studies will be needed to test this hypothesis.

\section{Conclusion}

In conclusion, the clinical performance characteristics of the First Sign ${ }^{\circledR}$ SARS-CoV-2 Antigen Test was observed to be comparable to the EUA RT-PCR comparator when testing individuals within the first 5 days of symptom onset. Study results indicate that during the initial phase of infection, when upper respiratory viral loads and viral shedding levels are typically highest [28], an observed anterior nasal 
swab self-collected by the patient and applied to the First Sign ${ }^{\circ}$ SARSCoV-2 Antigen Test may be as effective in detecting the presence of SARS-CoV-2 in symptomatic individuals as a EUA RT-PCR assay. Additionally, the ability to obtain a low-cost qualitative result within 15 minutes, combined with the simple anterior nasal self-sampling procedure, may dramatically increase testing efficiency while significantly reducing risk to health care providers by minimizing or eliminating the need for close contact with the patient.

\section{Ethics Statement}

This research was performed in accordance with Good Clinical Practice guidelines and the Declaration of Helsinki. This study was approved by Advarra Institute Review Board (Number: Pro00048222).

\section{Author Contributions}

JR and JW: study overall conception and design. WY and ER: study conduct, data analysis, and manuscript preparation. CP: study design and antigen test. AP: subject enrollment and antigen test.

\section{Funding}

This study was supported by industry funding from W.H.P.M., Inc.

\section{References}

1. World Health Organization (2020) WHO Coronavirus Disease (COVID-19) Dashboard. WHO, Geneva, Switzerland.

2. Corman VM, Landt O, Kaiser M, Molenkamp R, Meijer A, et al. (2020) Detection of 2019 novel coronavirus (2019-nCoV) by realtime RT-PCR. Euro Surveill 25: 2000045.

3. Iacobucci G (2020) COVID-19: Testing service wasn't prepared for increased demand, chief admits. BMJ 370: m3676.

4. James AE, Gulley T, Kothari A, Holder K, Garner K, et al. (2021) Performance of the BinaxNOW coronavirus disease 2019 (COVID-19) Antigen Card test relative to the severe acute respiratory coronavirus virus 2 (SARS-CoV-2) real-time reverse transcriptase polymerase chain reaction (rRT-PCR) assay among symptomatic and asymptomatic healthcare employees. Infect Control Hosp Epidemiol 1-3.

5. Tait RC, Hung A, Gardner RS (2019) Performance of the LumiraDx Platform INR Test in an Anticoagulation Clinic Point-of-Care Setting Compared With an Established Laboratory Reference Method. Clin Appl Thromb Hemost 25: 1076029619890423.

6. Young S, Taylor SN, Cammarata CL, Varnado KG, Roger-Dalbert C, et al. (2020) Clinical Evaluation of BD Veritor SARS-CoV-2 Point-ofCare Test Performance Compared to PCR-Based Testing and versus the Sofia 2 SARS Antigen Point-of-Care Test. J Clin Microbiol 59: e02338-e02420.

7. Mattiuzzi C, Henry BM, Lippi G (2020) Making sense of rapid antigen testing in severe acute respiratory syndrome coronavirus 2 (SARSCoV-2) diagnostics. Diagnosis (Berl) dx-2020-0131.

8. Dinnes J, Deeks JJ, Adriano A, Berhane S, Davenport C, et al. (2020) Rapid, point-of-care antigen and molecular-based tests for diagnosis of SARS-CoV-2 infection. Cochrane Database Syst Rev 8: CD013705.

9. Mojsoska B, Larsen S, Olsen DA, Madsen JS, Brandslund I, et al. (2021) Rapid SARS-CoV-2 Detection Using Electrochemical Immunosensor. Sensors 21: 1-11.

10. Okoye NC, Barker AP, Curtis K, Orlandi RR, Snavely EA, et al. (2021) Performance Characteristics of BinaxNOW COVID-19 Antigen Card for Screening Asymptomatic Individuals in a University Setting. J Clin Microbiol 03282-03320.
11. Raziq A, Kidakova A, Boroznjak R, Reut J, Öpik A, et al. (2021) Development of a portable MIP-based electrochemical sensor for detection of SARS-CoV-2 antigen. Biosens Bioelectron 178: 113029.

12. Salvagno GL, Gianfilippi G, Bragantini D, Henry BM, Lippi G (2021) Clinical assessment of the Roche SARS-CoV-2 rapid antigen test. Diagnosis (Berl).

13. Porte L, Legarraga $P$, Iruretagoyena $M$, Vollrath V, Pizarro G, et al. (2021) Evaluation of two fluorescence immunoassays for the rapid detection of SARS-CoV-2 antigen-new tool to detect infective COVID-19 patients. PeerJ 9: e10801.

14. Kobayashi R, Murai R, Asanuma K, Fujiya Y, Takahashi S (2021) Evaluating a novel, highly sensitive, and quantitative reagent for detecting SARS-CoV-2 antigen. J Infect Chemother 27: 800-807.

15. Gremmels $H$, Winkel BMF, Schuurman R, Rosingh A, Rigter NAM, et al. (2021) Real-life validation of the Panbio ${ }^{\mathrm{TM}}$ COVID-19 antigen rapid test (Abbott) in community-dwelling subjects with symptoms of potential SARS-CoV-2 infection. EClinicalMedicine 31: 100677.

16. Krüttgen A, Cornelissen CG, Dreher M, Hornef MW, Imöhl M, et al. (2021) Comparison of the SARS-CoV-2 Rapid antigen test to the real star Sars-CoV-2 RT PCR kit. J Virol Methods 288: 114024.

17. Rao SN, Manissero D, Steele VR, Pareja J (2020) A Systematic Review of the Clinical Utility of Cycle Threshold Values in the Context of COVID-19. Infect Dis Ther 9: 573-586.

18. Singanayagam A, Patel $M$, Charlett A, Lopez Bernal J, Saliba $V$, et al. (2020) Duration of infectiousness and correlation with RT-PCR cycle threshold values in cases of COVID-19, England, January to May 2020. Euro Surveill 25: 2001483.

19. Pekosz A, Parvu V, Li M, Andrews JC, Manabe YC, et al. (2021) Antigen-Based Testing but Not Real-Time Polymerase Chain Reaction Correlates With Severe Acute Respiratory Syndrome Coronavirus 2 Viral Culture. Clin Infect Dis ciaa1706.

20. Kohmer N, Toptan T, Pallas C, Karaca O, Pfeiffer A, et al. (2021) The Comparative Clinical Performance of Four SARS-CoV-2 Rapid Antigen Tests and Their Correlation to Infectivity In vitro. J Clin Med 10: 328.

21. Lauring AS, Hodcroft EB (2021) Genetic Variants of SARS-CoV-2What Do They Mean? JAMA 325: 529-531.

22. Tang JW, Tambyah PA, Hui DS (2020) Emergence of a new SARSCoV-2 variant in the UK. J Infect 82: e27-e28.

23. Li Q, Wu J, Nie J, Zhang L, Hao H, et al. (2020) The Impact of Mutations in SARS-CoV-2 Spike on Viral Infectivity and Antigenicity. Cell 182: 1284.e9-1294.e9.

24. Zhang W, Davis BD, Chen SS, Sincuir Martinez JM, Plummer JT, et al. (2021) Emergence of a Novel SARS-CoV-2 Variant in Southern California. JAMA e211612.

25. Zhu Y, Liu M, Zhao W, Zhang J, Zhang X, et al. (2005) Isolation of virus from a SARS patient and genome-wide analysis of genetic mutations related to pathogenesis and epidemiology from 47 SARSCoV isolates. Virus Genes 30: 93-102.

26. Grifoni A, Sidney J, Zhang Y, Scheuermann RH, Peters B, et al. (2020) A Sequence Homology and Bioinformatic Approach Can Predict Candidate Targets for Immune Responses to SARS-CoV-2. Cell Host Microbe 27: 671.e2-680.e2.

27. Oliveira SC, de Magalhaes MTQ, Homan EJ (2020) Immunoinformatic Analysis of SARS-CoV-2 Nucleocapsid Protein and Identification of COVID-19 Vaccine Targets. Front Immunol 11: 587615.

28. Yilmaz A, Marklund E, Andersson M, Nilsson S, Andersson LM, et al. (2021) Upper Respiratory Tract Levels of Severe Acute Respiratory Syndrome Coronavirus 2 RNA and Duration of Viral RNA Shedding Do Not Differ Between Patients With Mild and Severe/Critical Coronavirus Disease 2019. J Infect Dis 223: 15-18.

Citation: Yu W, Rao E, Wan DD, Patterson C, Wan DD, et al. (2021) A Prospective Study to Evaluate the Clinical Performance of A Novel Multitarget-Based Antigen Test on the Detection of Sars-Cov-2 in Symptomatic Population-Comparison to Nuclear Acid-Based Tests. J 